\title{
Nonlinear damped vibrations of planar discrete systems - numerical and experimental modelling
}

\author{
Peter Pavlov ${ }^{1 *}$ \\ ${ }^{1}$ University of Architecture, Civil Engineering and Geodesy, $1 \mathrm{Hr}$. Smirnenski B lvd., Sofia 1046, \\ Bulgaria.
}

\begin{abstract}
The consistently conducted analytical, numerical and experimental studies of nonlinear damped vibrations of planar discrete systems are presented in the paper. The combined methodology is applied to a horizontal vibrating system, consisting of two translational moving bodies connected by three springs. The non-linear nature of the damping is due to the dry friction forces accompanying the vibrating process. The mathematical model of the vibrating system is composed in a matrix form by the second order Lagrange equations. Numerical studies are realized in two ways. Firstly, in the Simulink environment, a simulation model was composed. Then, in the MATLAB environment, an animation model was developed using the third animation method offered by the programming system. The experimental studies were conducted by stand for study the small vibrations of discrete planar systems. The stand is part of the experimental equipment of the $\mathrm{Lab}$ for numerical and experimental dynamic modelling, UACEG, Sofia, Bulgaria. (www.dlab-uacg-bg.eu). All models - the dynamic model and its corresponding mathematical, simulation, animation and experimental model are open to additional bodies to obtain discrete vibrating systems with a larger number of degrees of freedom.
\end{abstract}

\section{Introduction}

The logical sequence of work, in studying any dynamic problem in the area of mechanical vibrations, is to conduct analytical, numerical and experimental research. The analytical studies are related to clarification of the dynamic model of the vibrating system, inference of the differential equations (DE), describing its motion and integration of the DE, when a precise analytical solution is possible. This is possible in linear DE, or linear systems with a small number of degrees of freedom (DOF). In the process of the numerical studies, the researcher visualizes the results of the exact analytical solution, or integrates DE and visualizes results, when an analytical solution is difficult to implement. The experimental studies are used to verify the results of the first two stages. Their performance is related to

* Corresponding author: pdp mech fhe@uacg.bg 
the design of an experimental model that fully corresponds to the dynamic model of the vibrating system.

An interesting problem of overcoming occurs often in an experimental study of free undamped vibrations of a standard discrete vibrating system. Even with the most precise experimental realization, after a certain period of time, the amplitudes of the vibrations decrease. The reason for this is the minimum friction forces that arise in the internal or external connections of the system.

Dry friction forces are resisting forces that slow the movement of the material objects as they are always directed in the opposite direction to the mutual velocity between them or their velocity, relative to the fixed elements. In the case of mechanical vibrations, which typically occur around the stable equilibrium position of the systems, the dry friction forces act as a damper, as they help to damping the vibrations.

To determine how the friction forces affect the vibrations of the material objects, a combined study of a discrete vibrating system with two degrees of freedom was conducted. Unlike the logical sequence of work, noted at the beginning of the Introduction, in the present study the dynamic model is based on an already constructed experimental model and corresponds not to a certain real system, but rather to the experimental model.

\section{Dynamic and numerical modelling of modified existing experimental model}

As a subject of investigation, a constructed stand for study the small vibrations of planar discrete systems $[1,2]$ is used. The stand allows experimental study of the standard types of vibrations - free and forced, undamped and damped vibrations. The problem mentioned in the introduction is most apparent in the study of free damped vibrations.

\subsection{Experimental model}

Photos of the constructed experimental configuration are shown on Fig. 1. and 2.

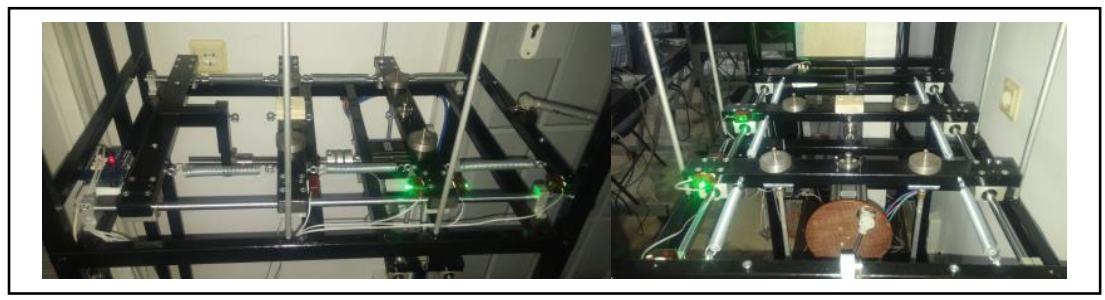

Figure 1. Photos of an existing experimental configuration.

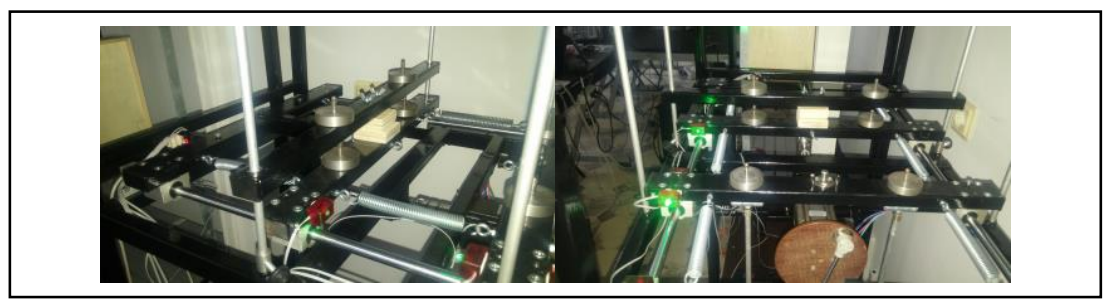

Figure 2. Photos of modified experimental model for study of damped vibrations, caused by dry friction forces. 
The experimental model, a major part of the experimental configuration, consists of two metal bodies, which move translationally on metal guides. The connection between the bodies and the guides is realized through special linear bearings. The elastic connections between the bodies and the fixed points are modeled by systems of two parallel springs, working on a tension. The model built into the stand construction allows the realization of damped vibrations by hydraulic damper on the second body.

To measure the displacements of the two bodies, a light system of lamps and photo resistors has been developed, which directs the numerical data to a computer configuration through a transformed Arduino controller.

The stand is modified by adding an additional body to press on one of the bodies. As a result of the impact, additional forces of dry friction occur in the horizontal movement of the body.

\subsection{Dynamic model}

The dynamic model, used in this study, corresponds to the modified experimental model for study of the free damped vibrations in the presence of dry friction forces. The two translationally moving bodies vibrate around the position of a stabile equilibrium, corresponding to unstressed springs. Dry friction force is applied to body 1, caused by additional applied body. The sliding friction coefficient is $\mu$. The model is shown on Fig.3.

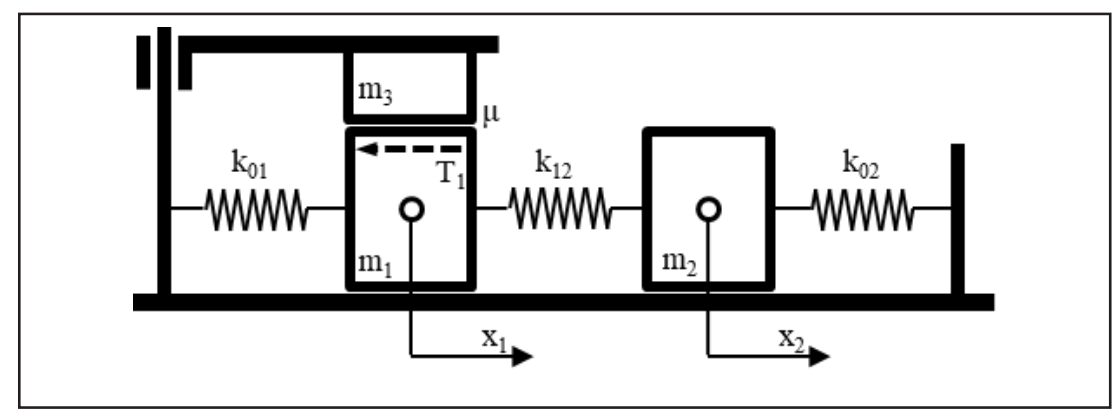

Figure 3. Dynamic model of Nonlinear damped vibrations of planar discrete systems.

\subsection{Mathematical model}

Differential equations, describing the free damped vibrations of the system, are derived from the second order Lagrange equations. The matrix form of the equations is as follows

$$
[\mathbf{M}]_{2 x 2} *\{\ddot{\mathbf{x}}\}_{2 x 1}+[\mathbf{C}]_{2 x 2} *\{\mathbf{x}\}_{2 x 1}=\{\mathbf{Q}\}_{2 x 1} .
$$

The mass and elastic matrices of equation (1) will have the form

$$
[\mathbf{M}]_{2 \times 2}=\left[\begin{array}{cc}
m_{1} & 0 \\
0 & m_{2}
\end{array}\right],[\mathbf{C}]_{2 \times 2}=\left[\begin{array}{cc}
k_{01}+k_{12} & -k_{12} \\
-k_{12} & k_{12}+k_{02}
\end{array}\right] .
$$

The dry friction force is included in the generalized force vector. The three vectors of Eq. (1) will be

$$
[\ddot{\mathbf{x}}]_{2 x 1}=\left[\begin{array}{c}
\ddot{x}_{1} \\
\ddot{x}_{2}
\end{array}\right],[\mathbf{x}]_{2 x 1}=\left[\begin{array}{l}
x_{1} \\
x_{2}
\end{array}\right],[\mathbf{Q}]_{2 x 1}=\left[\begin{array}{c}
-\mu * m_{3} * g * \operatorname{sign}\left(\dot{x}_{1}\right) \\
0
\end{array}\right]
$$




\subsection{Simulation model}

The simulation model is compiled in Simulink's graphical environment [3]. For easier tracking of the integration path, the model is based on a solution of Eq. (1) in terms of individual accelerations.

$$
\begin{aligned}
& \ddot{x}_{1}=-\frac{\mu * m_{3} * g}{m_{1}} * \operatorname{sign}\left(\dot{x}_{1}\right)-\frac{\left(k_{01}+k_{12}\right)}{m_{1}} * x_{1}+\frac{k_{12}}{m_{1}} * x_{2}, \\
& \ddot{x}_{2}=\frac{k_{12}}{m_{2}} * x_{1}-\frac{\left(k_{12}+k_{02}\right)}{m_{2}} * x_{2} .
\end{aligned}
$$

The simulation model developed in a single window is shown in Fig. 4.

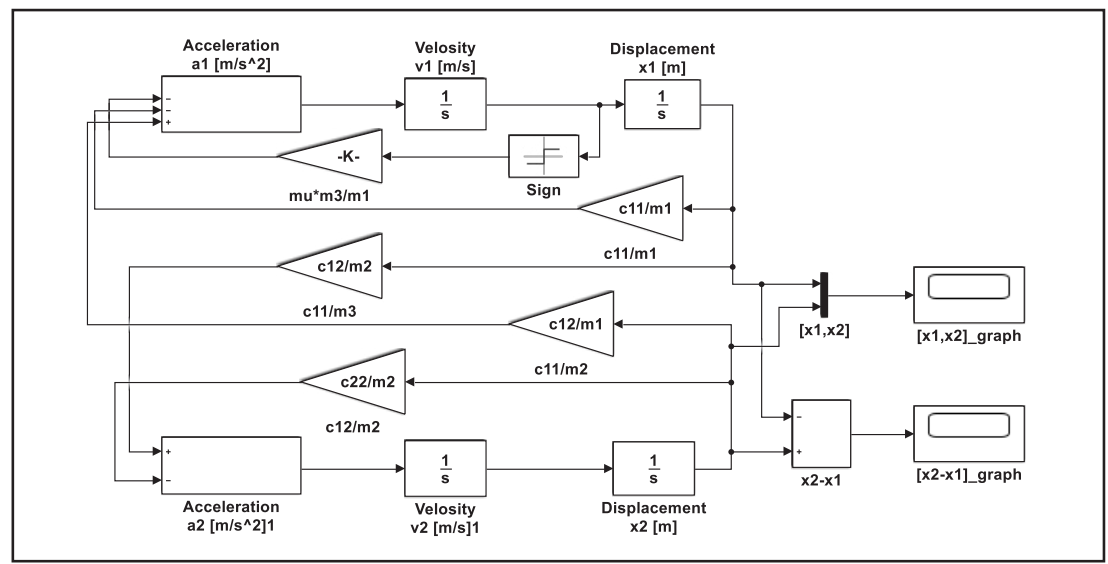

Figure 4. Simulation model of Nonlinear damped vibrations of planar discrete systems.

\subsection{Animation model}

A very attractive application, in study of vibrating processes, is the animation. In this study, a third animation method [4], offered by the MATLAB program, is used. The developed animation program also generates a video file whereby the movement of the vibrating system can also be monitored by hardware that does not have the software installed.

Animation models are composed of a large number of graphic elements - lines, rectangles, and others, that model the vibrating system. The animation is based on results obtained in Simulink. It is possible to implement integration of the DE, describing motion of the vibrating system directly into the animation program file.

The animation model of the vibrating system and a separate frame of the generated video file are shown in Fig. 5.

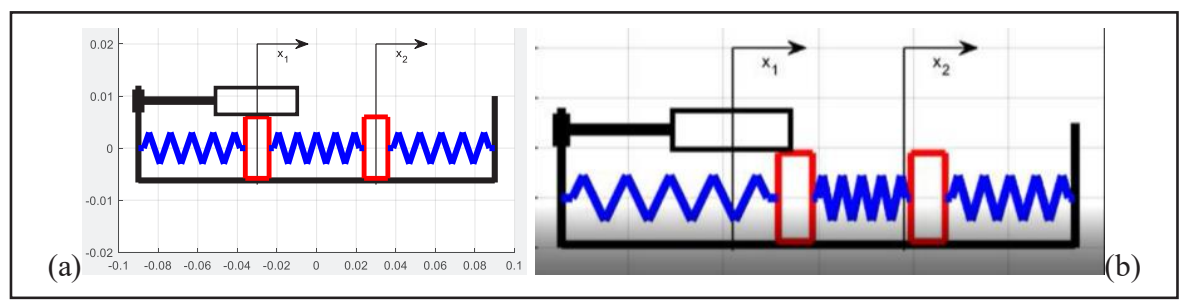

Figure 5. Animation model (a) and separate frame from the corresponded video file (b). 


\section{Results}

The varied, combined nature of the present study implies also variety in the nature of the results. Fig. 6 shows vibration of a planar discrete system in the absence and in the presence of dry friction forces while other parameters are kept the same. The values of the characteristics, shown in Fig. 3 are $m_{1}=1 \mathrm{~kg}, \mathrm{~m}_{2}=3 \mathrm{~kg}, \mathrm{~m}_{3}=1 \mathrm{~kg}, \mathrm{k}_{01}=100 \mathrm{~N} / \mathrm{m}, \mathrm{k}_{12}=200 \mathrm{~N} / \mathrm{m}$, $\mathrm{k}_{02}=200 \mathrm{~N} / \mathrm{m}, \mu=0.1$.

(a)

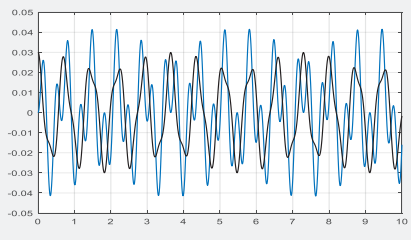

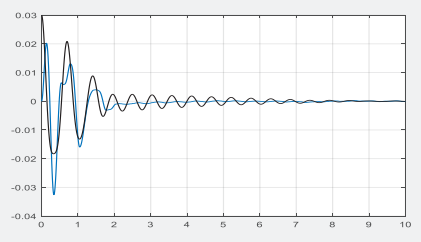

(b)

Figure 6. Free undamped (a) and nonlinear free damped (b) vibration in the presence of dry friction forces - numerical results. The blue line represents the first body and the black line - the second.

Even in the presence of a very low dry friction, the vibration of the first body fades quickly, but the vibration of the second body is slower.

Data from the numerical study allows experimental realization. The results of the experimental studies show good agreement with the numerical data. The difference, which in percent is about 5 , is due to the extra frictional forces that accompany the movement of the bodies in the experimental model - Fig. 7.

(a)

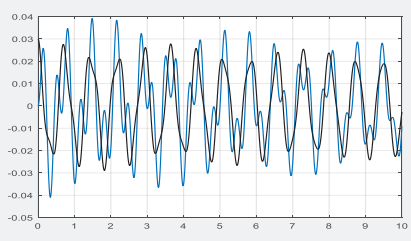

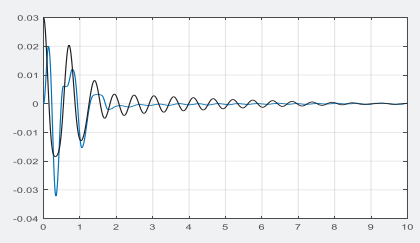

(b)

Figure 7. Free undamped (a) and nonlinear free damped (b) vibration in the presence of dry friction forces - experimental results. The blue line represents the first body and the black line - the second.

\section{Conclusion}

The created models (dynamic, mathematical, simulation, animation, experimental) are the base for the development of deep studies in the area of vibrations in the presence of dry friction forces. The results of the conducting various numerical and stand experiments allow a varied analysis of the influence of the dry friction forces on the vibrating systems.

Models are open and allow upgrading both in terms of degrees of freedom and impact on moving material objects.

\section{References}

[1] P. Pavlov. Annual of UACG, vol. 51, p. 59-64. (2018).

[2] P. Pavlov. Mechanics of the mashines, vol. 120, p. 38-41. (2018).

[3] A. Tyagi, MATLAB and Simulink for Engineers. Oxford university press (2012).

[4] W. Palm. Introduction to MATLAB for Engineers. McGray Hill Education, New York, (2011). 